\title{
Stackelberg Game for Product Renewal in Supply Chain
}

\author{
Yong Luo, Yamin Lou, and Shizhao Wang \\ School of Electrical Engineering, Zhengzhou University, Zhengzhou 450001, China \\ Correspondence should be addressed to Yong Luo; luoyong@zzu.edu.cn
}

Received 8 November 2012; Accepted 27 January 2013

Academic Editor: Xiaochen Sun

Copyright (C) 2013 Yong Luo et al. This is an open access article distributed under the Creative Commons Attribution License, which permits unrestricted use, distribution, and reproduction in any medium, provided the original work is properly cited.

\begin{abstract}
The paper studied the process of product renewal in a supply chain, which is composed of one manufacturer and one retailer. There are original product and renewal product in the supply chain. A market share shift model for renewal product was firstly built on a increment function and a shift function. Based on the model, the decision-making plane consisting of two variables was divided into four areas. Since the process of product renewal was divided into two stages, Stackelberg-Nash game model and Stackelbergmerger game model could be built to describe this process. The optimal solutions of product pricing strategy of two games were obtained. The relationships between renewal rate, cost, pricing strategy, and profits were got by numerical simulation. Some insights were obtained from this paper. Higher renewal rate will make participants' profits and total profit increase at the same margin cost. What is more important, the way of the optimal decision making of the SC was that RP comes onto the market with a great price differential between OP and RP.
\end{abstract}

\section{Introduction}

With the development of IT, the speed of product renewal process becomes faster and faster. This situation brings some new problems to traditional supply chain (SC), such as the dynamic nature of an SC and the relationship coordination between participants of an SC. The dynamic nature of an SC includes the dynamic variety of products; that is to say, the market requests SC to satisfy diverse needs of customer with the fastest speed, as well as the best quality, which calls for SC improving performance to adapt to the product variety. A valid way for SC enterprises to follow the variety of market is to renew their existing products, which can make full use of enterprise's existing resources and supply/distribution outlet of SC. Thus, SC will adapt to a variational market economically and quickly. Product renewal is an effective method that strengthens a SC enterprise's core competitiveness, and it will even impact on the survival of a enterprise. There are quite a few cases that enterprises collapse because of the mistakes of product renewal decision making, such as the failure of WANGAN Computer Corp.

Now, it is necessary to differentiate "renewal product (RP)" from "innovated product (IP)." IP's structure and principle are different from original product (OP), while RP's main structure and principle are the same as OP. Furthermore, RP has some appended components or upgraded functions. Thereby, RP is the renewal of existing product. At present, most researches are mainly focused on complete new product innovation, including management of product innovation process, optimization of product innovation investment, promotion of new product, and design of product innovation drive mechanism. Dereli et al. proposed a framework of the rapid response for innovative product development using reverse engineering approach [1]. Bourreau et al. studied the effects of modular design on firms' product innovation strategies and postinnovation competition in digital market [2]. Ju and Xiao built a model of product lifecycle evaluation based on context knowledge [3]. Wan et al. built a new product investment model [4]. Researches about product innovation based on SC frame have also emerged. Huo has studied the R\&D strategy in SC using game theory [5].

At present, the researches concerning product renewal have been carried out. Bass P. I. and Bass F. M. had proposed a new product expansion model and studied the process of multigeneration renewal products coming onto market $[6,7]$. $\mathrm{Wu}$ examined the reuse/redesign, quality, speed-to-market, 
and marketing decisions for two consecutive generations of a multicomponent modular product, utilizing stylized models [8]. Druehl et al. developed a model to gain insight into which factors drive the pace of product updates [9]. Huang and Ling studied the tracks of product update of ICT enterprises [10]. Koca et al. studied the product rollover strategy decision, where a firm decides whether to phase out an old generation product to be replaced by a new with either a dual or single roll [11]. Quan analyzed the relationship of the optimal launch time with parameters in the process of introducing a renewal product [12].

The other focus of product renewal is on the marketing process of renewal product, especially pricing decision. Luo and Tu studied a price decision problem for product renewal supply chain based on Nash game [13]. Wei studied the optimal decision of inventory and pricing in a supply chain, in which there are original products and renewal products [14]. But the work of product renewal is far less than enough.

The RP and OP are virtually differentiated products. When they are on sale at the same market, they can substitute for each other. Compared with common differentiated products, the substitution of RP for OP is unidirectional and partial. Therefore, the coming of RP onto market will cause some influences on OP market. At the same time, each entity in SC has independent profit. Therefore, the coordination of SC will become much more complex if there are RP and OP in the SC. A potent tool to coordinate SC is price. The fluctuation of price will make supply and demand tend to an equilibrium. On the other hand, price's fluctuation can make the profit of each entity in SC distributed reasonably, so as to achieve the aim of coordination of SC. Therefore pricing strategy is the main content of the paper.

The SC including OP and RP is called product renewal $\mathrm{SC}$. The change of market share caused by product renewal in the SC is studied in this paper. When there is a RP in SC, the enterprises in SC will make strategies for both of OP and RP to achieve an equilibrium of enterprises' profits. Compared with other researches, this paper firstly studied Stackelberg game for product renewal, aiming at solving complex decision-making question in actual renewal SC, and obtained some novel insights.

The rest of this paper is organized as follows. Product renewal model is formulated in Section 2. In Section 3, we provide a Stackelberg-Nash Game based on product renewal model. Stackelberg-merger game is played in Section 4, and numerical examples are provided in Section 5. Finally, we conclude our paper in Section 6.

\section{Model Description}

2.1. Assumption. Consider a two-stage SC which is composed of one manufacturer and one retailer. First, suppose the manufacturer produces product $A$ and sells it in market via the retailer. After a period of renewal process, the manufacturer develops product $A$ 's renewal product, $B$, and sells it with product $A$ in market via the retailer.

In order to fully describe the model of the SC, we state the following hypotheses.

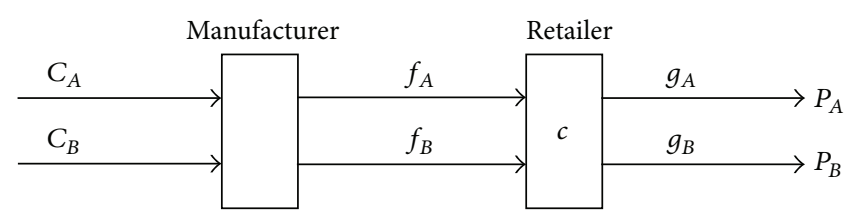

FIgURE 1: The structure of renewal products SC.

(i) The retailer is in a monopoly position; that is, only the retailer sells $A$ and $B$ in market.

(ii) Suppose that the consumer's repurchase is possible and each time one customer only buys one product. When there is an RP, some customers of OP may shift to buying RP in other words, there is repetition purchase for the products studied in this paper, such as toys, and small electric appliance equipment.

(iii) There is no stochastic demand in the market, so the demand is only decided by price.

(iv) Inventory is not considered.

(v) The manufacturer's productivity is sufficient enough to satisfy demand.

(vi) There is no fixed cost for unit product, and margin production cost is an invariant for manufacturer.

(vii) The product renewal generally causes its performance promotion.

The SC model of this paper is as follows: there is one manufacturer and one retailer in SC. Suppose that the manufacturer produces products $A$ and $B$ at marginal costs $C_{A}$ and $C_{B}$, respectively, and then distributes them at the wholesale price of $f_{A}$ and $f_{B}$ to retailer. Based on the wholesale price, a price markup $g_{A}$ and $g_{B}$ is, respectively, added by retailer to product $A$ and $B$ to form the market retail price: $P_{i}=$ $g_{i}+f_{i}$. Retailer's selling cost is $c$ per unit. The product renewal SC including one manufacturer and one retailer that sells combination products is shown in Figure 1.

2.2. Market Model. Product renewal rate is used to measure the change of product performance caused by product renewal. Combined with price and performance price ratio $\left(E_{i j}=k P_{j} / P_{i}\right)$, product renewal rate can be used to forecast the market share change. In fact, what is focused on is not performance value, but the ratio of OP's performance value to RP's [15], namely renewal rate $k(k>1)$.

The RP marketing process can be divided into two stages:

In Stage 1. there is only OP in market. Supposing the OP price of $A$ is $P_{A}$, according to [15], the market demand for $A$ is

$$
T=a-b P_{A}
$$

In expression (1), a means market scale, the absolute value of $b$ denotes price flexibility, and $b>0$.

In Stage 2. RP comes onto the market and competes with OP. If RP comes onto the market, it will cause 
several changes in the market: (1) RP will stimulate new consumption demand because of its appended or upgraded functions, which makes the latent customer quantity of the total products market enlarge, and the increment is $\Delta T . \Delta T$ is related to original market demand $T$ and a decreasing function of RP price, while an increasing function of renewal rate $k$. (2) Customers have the tendency to purchase RP. In addition, the performance price ratio of RP is generally higher than that of OP. So some OP's customers will shift to buying RP. The number of shift customers is marked as $T_{s}$. (3) This will cause OP's price to be adjusted to market.

Definition 1. There exists an OP $A$ and its RP $B$ in the market. Suppose that the demand of $A$ is $T$, and the product renewal rate is $k$. Then $\Delta T$ satisfies the following function:

$$
\Delta T=w_{1} T\left(k-w_{2} P_{B}\right)
$$

where $w_{1}$ is the influence coefficient of $T$ and $0<w_{1}<1 . w_{2}$ is the influence coefficient of RP's price on its market increment, and $w_{2} \ll 1$.

From $\Delta T=0$ and $T=0, P_{B 1}=k / w_{2}$ and $P_{A 1}=a / b$ can be got. If $P_{A}>P_{A 1}$, OP's demand will be zero, which leads $\Delta T$ to be zero. If $P_{B}>P_{B 1}$, no matter how great OP's demand is, demand increment $\Delta T$ will be equal to zero.

Theorem 2. The market shift function $T_{s}$ relates to $O P$ price, $R P$ price, and OP demand. $T_{s}$ has the following features.

(i) If $P_{B} \leq P_{A}, T_{s}=T$, that is, all of the OP demand will shift to RP. So OP will have to exit from the market, and $R P$ will hold the market.

(ii) Existing $P_{B 0}>P A$, if $P_{B} \geq P_{B 0}, T_{s}=0 . P_{B 0}$ can be treated as the zero point of market shift.

(iii) If $P_{A}<P_{B}<P_{B 0}, T_{s}$ will be a monotone decreasing function of $P_{B}$, namely, $\partial T_{s} / \partial P_{B}<0$.

(iv) If $P_{A}<P_{B}<P_{B 0}, T_{s}$ is to be a monotone increasing function of $P_{A}$, namely, $\partial T_{s} / \partial P_{A}>0$.

(v) With the increase of $k, P_{B 0}$ increases correspondingly. $T_{s}$ is an increasing function of $k$, namely, $\partial T_{s} / \partial k>0$.

(vi) Let $\Delta T=0$, and the corresponding price is $P_{B 1}$, which is the upper limit of RP price.

(vii) Let $T_{s}=0$, and there is still demand increment caused by $R P$; that is to say, on any condition, $P_{B 0}<P_{B 1}$.

(viii) $T_{s}$ is a direct proportion increasing function with $T$.

From Theorem 2, given $P_{A}$, the laws of changes of $T_{s}$ and $\Delta T$ can be got with the variety of $P_{B}$, as shown in Figure 2. The specific shape of this figure is changing with the variety of $P_{A}$.

According to Figure 2, the shift function $T_{s}$ in $\left[0, P_{A}\right]$ is

$$
T_{s 1}=T
$$

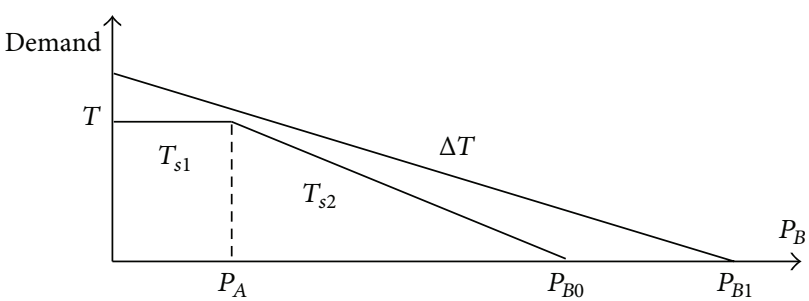

FIgURE 2: The changes of $T_{s}$ and $\Delta T$ with the variety of $P_{B}$.

Definition 3. According to Figure 2, in $\left[P_{A}, P_{B 0}\right]$, there exist an OP $A$ and its RP $B$; suppose the prices of $A, B$ are $P_{A}, P_{B}$, respectively, and the demand of $A$ is $T$. Then, $T_{s 2}$ satisfies the following function:

$$
T_{s 2}\left(P_{A}, P_{B}\right)=w_{3} T\left(k w_{4} P_{A}-P_{B}\right),
$$

where $w_{3}$ is the influence coefficient of $P_{A}$ and $P_{B}, 0<w_{1}<1$, $w_{4}$ is the influence coefficient of $P_{A}$, and $w_{4}>1 / k$.

The functions (3) and (4) meet eight features listed previewed, and can be used as the paper's model base. From the previous analysis, the shift function includes two portions, so it can be expressed as follows:

$$
T_{s}= \begin{cases}T & P_{B} \leq P_{A}, \\ {\left[w_{3} T\left(k w_{4} P_{A}-P_{B}\right)\right]^{+}} & P_{B}>P_{A} .\end{cases}
$$

Letting $T_{s}=0$ and solve (5), the zero point of market shift function $P_{B 0}$ can be got:

$$
P_{B 0}=k w_{4} P_{A}
$$

With the increase of $k, P_{B 0}$ obviously becomes higher. Therefore, when RP comes onto the market, the total demand will change. The market share of RP $B$ is

$$
T_{B}=\Delta T+T_{s},
$$

and OP $A$ is

$$
T_{A}=T-T_{s}
$$

In addition, product renewal will make marginal production cost change from $C_{A}$ to $C_{B}$.

Figure 2 is a variety chart of shift demand $T_{s}$ in the situation that $P_{A}$ is given, so the shift demand $T_{s}$ actually depends on $P_{A}$ and $P_{B}$ simultaneously. Accordingly, a threedimensional coordinate can be built, $P_{A}-P_{B}-T_{s}$, as shown in Figure 3, and axis $T_{s}$ is shift demand. Plane $P_{A}-P_{B}$ is divided into the following four areas by six lines in Figure 3:

Area I: the area surrounded by axis $P_{A}$ and lines $P_{A}=$ $P_{B}$ and $P_{A}=a / b$,

Area II: the area surrounded by lines $P_{A}=P_{B}, P_{A}=$ $a / b$ and $P_{B}=k w_{4} P_{A}$, 


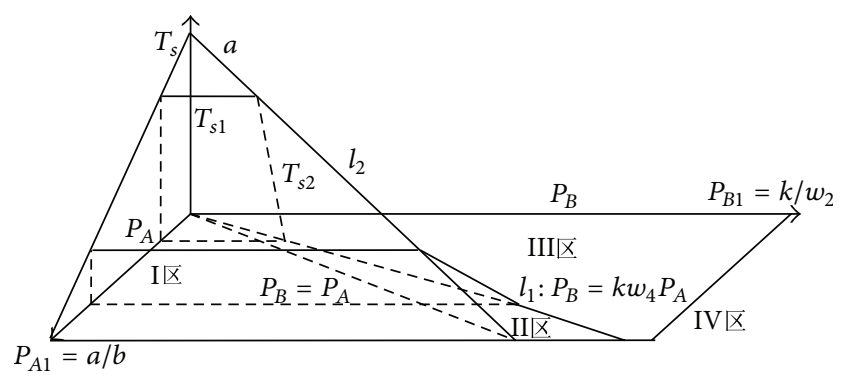

Figure 3: The change of $T_{s}$ with the variety of $P_{B}$ and $P_{A}$.

TABLE 1: The table of area's shift function and increment function.

\begin{tabular}{lcc}
\hline & $T_{s}\left(P_{A}, P_{B}\right)$ & $\Delta T\left(P_{A}, P_{B}\right)$ \\
\hline Area I & $T\left(P_{A}\right)$ & $w_{1} T\left(P_{A}\right)\left(k-w_{2} P_{B}\right)$ \\
Area II & $w_{3} T\left(P_{A}\right)\left(k w_{4} P_{A}-P_{B}\right)$ & $w_{1} T\left(P_{A}\right)\left(k-w_{2} P_{B}\right)$ \\
Area III & 0 & $w_{1} T\left(P_{A}\right)\left(k-w_{2} P_{B}\right)$ \\
Area IV & 0 & 0 \\
\hline
\end{tabular}

Area III: the area surrounded by axis $P_{B}$ and lines $P_{B}=$ $k w_{4} P_{A}, P_{A}=a / b$ and $P_{B}=k / w_{2}$,

Area IV: the areas excepting area I, II, and III.

In area $\mathrm{I}$, Letting $P_{A}=0, T_{s}$ 's maximum value $a$ can be obtained from (1) and (2). Given $P_{A}$, the line $T_{s 1}$ will be parallel to plane $P_{A}-P_{B}$, and it is clear that the slope of line $T_{s 1}$ is $k_{T s 1}=0$. From (3), with the increasing of $P_{A}, T$ will become small. Meanwhile, $T_{s 1}$ will become small. If $P_{A}=a / b$, $T=T_{s 1}=0$. Therefore, $T_{s}$ will be a sloped plane in area I.

In area II, given $P_{A}, k_{T s 2}=-w_{3} T<0$ can be obtained from (5). The line $T_{s 2}$ will become smoother and smoother with the increase of $P_{A}$ and $k_{T s 2}$. If $P_{A}=a / b, k_{T s 2}=T_{s 2}=$ 0 . Letting $T_{s 2}=0$ in (4), a line $l_{1}: P_{B}=k w_{4} P_{A}$ in $P_{A}-P_{B}$ plane can be got. The line linking maximal $T_{s}$ point $(0,0, a)$ to point $(a / b, a / b, 0)$ is a $T_{s}$ line named $l_{2}$. The lines linking the points at which line $l_{1}$ or $l_{2}$ intersects with $T_{s 2}$ constitute $T_{s}$ complex curving surface; in other words, $T_{s}$ in area II is a complex curving surface.

In area III, $P_{B}>k w_{4} P_{A}, T_{s}=0$ can be got from (5).

Considering $T_{s}$ function's feature (vii), it is obvious that market increment $\Delta T>0$ in area I, II, and III.

In area IV, $P_{A}>a / b$ or $P_{B}>k / w_{2}, \Delta T=T_{s}=0$. OP and $\mathrm{RP}$ do not come onto the market in this area.

The shift functions and increment functions in different areas are shown in Table 1.

According to the feature (vii) of shift function, the zero point of increment function is bigger than that of shift function, namely, $P_{B 1}>P_{B 0}=k w_{4} P_{A}$. From expression (1), it is known that the maximum of $P_{A}$ is $a / b$. So it requires $b>$ $a w_{2} w_{4}$ to meet the seventh feature of shift function. Based on the previous analysis, the retailer's and manufacturer's profits including the profits of $\mathrm{OP}$ and $\mathrm{RP}$ are, respectively, as follows:

$$
\begin{gathered}
\pi_{r}=\left(T_{s}+\Delta T\right)\left(g_{B}-c\right)+\left(T-T_{s}\right)\left(g_{A}-c\right), \\
\pi_{m}=\left(\Delta T+T_{s}\right)\left(f_{B}-C_{B}\right)+\left(T-T_{s}\right)\left(f_{A}-C_{A}\right)-C_{g} .
\end{gathered}
$$

Where $C_{g}$ is the fixed cost of the manufacturer. According to the price relationship between RP and OP in different areas, the profit functions can be shown as follows.

(i) In area I, $P_{B} \leq P_{A}, \Delta T>0$ and $T_{s}=T>0$. The market share of RP $B$ is $\Delta T+T_{s}$, and OP $A$ is zero. The profits of retailer and manufacturer are

$$
\begin{aligned}
\pi_{r}\left(g_{A}, g_{B}\right)= & T\left(1+w_{1}\left(k-w_{2} P_{B}\right)\right)\left(g_{B}-c\right) \\
= & \left(a-b\left(f_{A}+g_{A}\right)\right) \\
& \times\left(1+w_{1}\left(k-w_{2}\left(f_{B}+g_{B}\right)\right)\right)\left(g_{B}-c\right), \\
\pi_{m}\left(f_{A}, f_{B}\right)= & T\left(1+w_{1}\left(k-w_{2} P_{B}\right)\right)\left(f_{B}-C_{B}\right)-C_{g} \\
= & \left(a-b\left(f_{A}+g_{A}\right)\right) \\
& \times\left(1+w_{1}\left(k-w_{2}\left(f_{B}+g_{B}\right)\right)\right)\left(f_{B}-C_{B}\right)-C_{g} .
\end{aligned}
$$

(ii) In area II, $P_{A} \leq P_{B} \leq P_{B 0}, \Delta T>0$ and $T_{s}>T>0$. The market share of RP $B$ is $\Delta T+T_{s}$, and OP $A$ is $T-T_{s}$. The profits of retailer and manufacturer are

$$
\begin{aligned}
\pi_{r}\left(g_{A}, g_{B}\right)=T\left\{\left(w_{1}\left(k-w_{2} P_{B}\right)+w_{3}\left(k w_{4} P_{A}-P_{B}\right)\right)\right. \\
\quad \times\left(g_{B}-c\right)+\left(1-w_{3}\left(k w_{4} P_{A}-P_{B}\right)\right) \\
\left.\quad \times\left(g_{A}-c\right)\right\} \\
=\left(a-b\left(f_{A}+g_{A}\right)\right) \\
\times\left\{\left(w_{1}\left(k-w_{2}\left(f_{B}+g_{B}\right)\right)\right.\right. \\
\quad+w_{3}\left(k w_{4}\left(f_{A}+g_{A}\right)\right. \\
\left.\left.\quad-\left(f_{B}+g_{B}\right)\right)\right)\left(g_{B}-c\right) \\
\quad+\left(1-w_{3}\left(k w_{4}\left(f_{A}+g_{A}\right)-\left(f_{B}+g_{B}\right)\right)\right) \\
\left.\quad \times\left(g_{A}-c\right)\right\}, \\
\pi_{m}\left(f_{A}, f_{B}\right)=T\left\{\left(w_{1}\left(k-w_{2} P_{B}\right)+w_{3}\left(k w_{4} P_{A}-P_{B}\right)\right)\left(f_{B}-C_{B}\right)\right. \\
\left.+\left(1-w_{3}\left(k w_{4} P_{A}-P_{B}\right)\right)\left(f_{A}-C_{A}\right)\right\}-C_{g} \\
=\left(a-b\left(f_{A}+g_{A}\right)\right) \\
\times\left\{\left(w_{1}\left(k-w_{2}\left(f_{B}+g_{B}\right)\right)\right.\right. \\
\quad+w_{3}\left(k w_{4}\left(f_{A}+g_{A}\right)\right. \\
\quad \\
\left.\left.\left.\quad+\left(1-f_{B}+g_{B}\right)\right)\right)\left(k f_{B}\left(f_{A}+g_{A}\right)-\left(f_{B}+g_{B}\right)\right)\right) \\
\left.\quad \times\left(f_{A}-C_{A}\right)\right\}-C_{g} . \\
\end{aligned}
$$


(iii) In area III, $P_{B 0} \leq P_{B} \leq P_{B 1}, \Delta T>0$ and $T_{s}=0$. RP B's market share is $\Delta T$, and OP $A$ 's market share is $T$. The profits of retailer and manufacturer are

$$
\begin{aligned}
\pi_{r}\left(g_{A}, g_{B}\right)= & T\left\{w_{1}\left(k-w_{2} P_{B}\right)\left(g_{B}-c\right)+g_{A}-c\right\} \\
= & \left(a-b\left(f_{A}+g_{A}\right)\right) \\
& \times\left\{w_{1}\left(k-w_{2}\left(f_{B}+g_{B}\right)\right)\left(g_{B}-c\right)+g_{A}-c\right\}, \\
\pi_{m}\left(f_{A}, f_{B}\right)= & T\left\{w_{1}\left(k-w_{2} P_{B}\right)\left(f_{B}-C_{B}\right)+f_{A}-C_{A}\right\}-C_{g} \\
= & \left(a-b\left(f_{A}+g_{A}\right)\right) \\
& \times\left\{w_{1}\left(k-w_{2}\left(f_{B}+g_{B}\right)\right)\right. \\
& \left.\times\left(f_{B}-C_{B}\right)+f_{A}-C_{A}\right\}-C_{g} .
\end{aligned}
$$

(iv) In area IV, $P_{B}>P_{B 1}$ or $P_{A}>P_{A 1}, \Delta T=T_{s}=0$. The prices of $\mathrm{OP}$ and $\mathrm{RP}$ are so high that it could not come onto the market. It is not necessary to consider this situation.

\section{Stackelberg-Nash Game}

Product renewal process can be regarded as two stages. First, manufacturer and retailer make their optimal decisions for OP $A$, respectively, and synchronously. After OP A comes onto market, RP $B$ is developed by the manufacturer and comes onto market, too. In this condition, both OP $A$ and RP $B$ are sold in the same market. Then the manufacturer and retailer make their optimal decisions for RP $B$, respectively, and synchronously. The optimal decision-making process is also divided into two stages. The two-stage games constitute a Stackelberg-Nash game, which is the real process of product renewal game.

In Stage 1: There is a decision for product $A$. The manufacturer and the retailer play a Nash game. The manufacturer makes a strategic decision of $f_{A}$, and the retailer makes a strategic decision of $g_{A}$.

In Stage 2: There is a decision for product $B$. The manufacturer and the retailer also play a Nash game. The manufacturer makes a strategic decision of $f_{B}$, and the retailer makes a strategic decision of $g_{B}$.

The game solving process is divided into three steps. Following three steps, the optimal solution of product renewal Stackelberg-Nash game can be derived.

In Step 1, given $f_{A}$ and $g_{A}$, according to the Nash equilibrium of the manufacturer and retailer profit functions, the optimal reaction function of $f_{B}\left(f_{A}, g_{A}\right)$, $g_{B}\left(f_{A}, g_{A}\right)$ or the optimal value of $g_{B}$ and $f_{B}$ can be obtained from $\partial \pi_{r} / \partial g_{B}=0, \partial \pi_{m} / \partial f_{B}=0$.

In Step 2, put the optimal reaction function or the optimal value into the profit functions of Step 1, and the optimal solution $f_{A}{ }^{*}$ and $g_{A}{ }^{*}$ can be obtained from the Nash equilibrium of the manufacturer and retailer profit functions in Step 1.
In Step 3, letting $f_{A}{ }^{*}$ and $g_{A}{ }^{*}$ substitute $f_{A}$ and $g_{A}$ in the $f_{B}\left(f_{A}, g_{A}\right)$ and $g_{B}\left(f_{A}, g_{A}\right)$ of Step 2, the optimal value of $f_{B}{ }^{*}$ and $g_{B}{ }^{*}$ can be obtained.

Theorem 4. In Stackelberg-Nash game, in areas I, II, and III, given $f_{A}$ and $g_{A}, \pi_{r}$ is a convex function of $g_{B}$, and $\pi_{m}$ is convex functions of $f_{B}$.

Proof. In area I

$$
\begin{aligned}
& \frac{\partial \pi_{r}\left(g_{A}, g_{B}\right)}{\partial g_{B}}=\left(a-b\left(f_{A}+g_{A}\right)\right) \\
& \times\left(-2 w_{1} w_{2} g_{B}+w_{1} w_{2}\left(c-f_{B}\right)+w_{1} k+1\right), \\
& \frac{\partial \pi_{m}\left(f_{A}, f_{B}\right)}{\partial f_{B}}=\left(a-b\left(f_{A}+g_{A}\right)\right) \times\left(-2 w_{1} w_{2} f_{B}+w_{1} w_{2}\left(C_{B}-g_{B}\right)+w_{1} k+1\right), \\
& \frac{\partial \pi_{r}^{2}\left(g_{A}, g_{B}\right)}{\partial g_{B}^{2}}=-2 w_{1} w_{2}\left(a-b\left(f_{A}+g_{A}\right)\right)<0, \\
& \frac{\partial \pi_{m}^{2}\left(f_{A}, f_{B}\right)}{\partial f_{B}^{2}}=-2 w_{1} w_{2}\left(a-b\left(f_{A}+g_{A}\right)\right)<0 .
\end{aligned}
$$

Therefore, $\pi_{r}$ is a convex function of $g_{B}$, and $\pi_{m}$ is a convex function of $f_{B}$. The proofs in II and III are the same as area $\mathrm{I}$.

Theorem 5. The Stackelberg-Nash decision-making process can be divided into the following three situations.

(i) In area I, there is no Stackelberg-Nash equilibrium.

(ii) In area II, there exists only one optimal pricing strategy for Stackelberg-Nash game.

(iii) In area III, there exists only one optimal pricing strategy for Stackelberg-Nash game.

Proof. (i) In area I, according to (10), based on Theorem 4 and Step 1, the optimal value of $g_{B}$ and $f_{B}$ can be derived as follows:

$$
\begin{aligned}
g_{B}{ }^{*} & =\frac{\left(1+w_{1} k+3 w_{1} w_{2}\left(2 c-C_{B}\right)\right)}{\left(3 w_{1} w_{2}\right)}, \\
f_{B}{ }^{*} & =\frac{\left(1+w_{1} k+3 w_{1} w_{2}\left(2 C_{B}-c\right)\right)}{\left(3 w_{1} w_{2}\right)} .
\end{aligned}
$$

From the supply chain's structure, it is obvious that $g_{B}{ }^{*}+$ $f_{B}{ }^{*}=P_{B}{ }^{*}$. Because $P_{B}{ }^{*}$ is greater than $P_{A 1}$ (from Theorem 6), considering the condition of area $\mathrm{I}, P_{B} \leq P_{A}$, the conclusion that $P_{B}{ }^{*}$ is out of area I can be drawn. Accordingly, there is no Nash equilibrium in area I.

(ii) In area II, $P_{A} \leq P_{B} \leq P_{B 0}$. According to (11), based on Theorem 4 and Step 1, the optimal reaction 
$\left(f_{B}\left(f_{A}, g_{A}\right), g_{B}\left(f_{A}, g_{A}\right)\right)$ of the manufacturer and retailer can be got as follows:

$$
\begin{aligned}
f_{B}\left(f_{A}, g_{A}\right)=( & w_{3}\left(k w_{4}+2\right) f_{A}+w_{3}\left(k w_{4}-1\right) g_{A} \\
& +w_{1} k+2 w_{3}\left(C_{B}-C_{A}\right) \\
& \left.+w_{1} w_{2}\left(2 C_{B}-c\right)\right) /\left(3 w_{3}+3 w_{1} w_{2}\right), \\
g_{B}\left(f_{A}, g_{A}\right)=( & w_{3}\left(k w_{4}-1\right) f_{A}+w_{3}\left(k w_{4}+2\right) g_{A} \\
& +w_{1} k+w_{3}\left(C_{A}-C_{B}\right) \\
& \left.+w_{1} w_{2}\left(2 c-C_{B}\right)\right) /\left(3 w_{3}+3 w_{1} w_{2}\right) .
\end{aligned}
$$

From Step 2, two binary cubic equations can be got. Because the analytic solution of the equations cannot be found, the numerical method can be used to find the optimal approximate solution $\left(f_{A}{ }^{*}, g_{A}{ }^{*}\right)$. From Step 3, the optimal of $\left(f_{B}{ }^{*}, g_{B}{ }^{*}\right)$ can be got.

(iii) In area III, $P_{B 0} \leq P_{B} \leq P_{B 1}$. According to (12), based on Theorem 4 and Step 1, the optimal values of $g_{B}$ and $f_{B}$ of $\pi_{r}$ and $\pi_{m}$ are as follows:

$$
\begin{aligned}
& g_{B}{ }^{*}=\frac{\left(k+w_{2}\left(2 c-C_{B}\right)\right)}{\left(3 w_{2}\right)}, \\
& f_{B}{ }^{*}=\frac{\left(k+w_{2}\left(2 C_{B}-c\right)\right)}{\left(3 w_{2}\right)} .
\end{aligned}
$$

From Step 2, the optimal values $g_{A}{ }^{*}$ and $f_{A}{ }^{*}$ of $\pi_{r}$ and $\pi_{m}$ can be got as follows:

$$
\begin{aligned}
g_{A}{ }^{*}=-( & w_{1} b\left(k-w_{2} C_{B}\right)^{2}+2 w_{1} w_{2} b c\left(w_{2} C_{B}-1\right)+w_{1} b c^{2} w_{2}^{2} \\
& \left.+9 b w_{2}\left(C_{A}-2 c\right)-9000 w_{2}\right) /\left(27 w_{1} b\right) \\
f_{A}^{*}=-( & w_{1} b\left(k-w_{2} C_{B}\right)^{2}+2 w_{1} w_{2} b c\left(w_{2} C_{B}-1\right)+w_{1} b c^{2} w_{2}^{2} \\
& \left.+9 b w_{2}\left(c-2 C_{A}\right)-9000 w_{2}\right) /\left(27 w_{1} b\right)
\end{aligned}
$$

Theorem 6. The value of Nah equilibrium for area $I, P_{B}{ }^{*}$, is out of area $I$.

Proof. From the supply chain structure, it is obvious that: $g_{B}{ }^{*}+f_{B}{ }^{*}=P_{B}{ }^{*}$ :

$$
P_{B}^{*}=g_{B}^{*}+f_{B}^{*}=\frac{\left(2+2 w_{1} k\right)}{\left(3 w_{1} w_{2}\right)}+C_{B}-c .
$$

Because of $0<w_{1}<1$ and $k>1$,

$$
P_{B}^{*} \geq \frac{(2+2 k)}{\left(3 w_{2}\right)}+C_{B}-c \geq \frac{4}{\left(3 w_{2}\right)}+C_{B}-c,
$$

whereas $w_{2} \ll 1$,

$$
P_{B}^{*} \geq P_{A 1}=\frac{a}{b}
$$

It is clear that the value of Nah equilibrium, $P_{B}{ }^{*}$, is greater than $P_{A 1}$. Since $P_{A 1}$ is the maximum of $P_{A}$ in area $\mathrm{I}$, considering the condition of area $\mathrm{I}, P_{B} \leq P_{A}$, the value of Nah equilibrium, $P_{B}{ }^{*}$, does not satisfy this condition. So $P_{B}{ }^{*}$ is out of area I.

\section{Stackelberg-Merger Game}

In the decision-making process of Stackelberg-Nash game mentioned previously, if the manufacturer and retailer constitute a manufacturing and sales league to maximize the SC profit, that is, there is no retailer, and manufacturer sells its product in market directly, the product renewal StackelbergNash decision-making process will become a Stackelbergmerger decision-making process. This process can be divided into two stages, and this process will play a Stackelbergmerger game. In this condition, the league which consists of the manufacturer and the retailer can be regarded as a enterprise, because the league's decision variables are the retail price $P_{i}$ of OP and RP $\left(f_{i}\right.$ and $g_{i}$ can be regarded as the transfer price of the manufacturer and retailer profits distribution).

In Stage 1, the league sells OP $A$ and makes a strategic decision of $P_{A}$.

In Stage 2, the league sells OP $A$ and RP $B$ at the same time, and the league makes strategic decisions of $P_{B}$ and $P_{A}$.

The solving process of Stackelberg-merger game is divided into three steps, and by three steps the optimal solution of product renewal Stackelberg-merger game can be derived.

In Step 1, OP retail price $P_{A}$ is given; the optimal reaction function $P_{B}\left(P_{A}\right)$ or the optimal value of $P_{B}$ can be obtained from $\partial \pi / \partial P_{B}=0$.

In Step 2, put the optimal reaction function $P_{B}\left(P_{A}\right)$ or the optimal value $P_{B}{ }^{*}$ into the first stage; the optimal pricing $P_{A}{ }^{*}$ can be obtained from $\partial \pi / \partial P_{A}=0$.

In Step 3, put $P_{A}{ }^{*}$ into the optimal reaction function $P_{B}\left(P_{A}\right)$; the optimal pricing $P_{B}{ }^{*}$ can be derived.

Theorem 7. In Stackelberg-merger game, in areas I, II, and III, given $P_{A}, \pi$ is a convex function of $P_{B}$.

Proof. In area I

$$
\begin{aligned}
\frac{\partial \pi\left(P_{A}, P_{B}\right)}{\partial P_{B}}= & \left(a-b P_{A}\right) \\
& \times\left(-2 w_{1} w_{2} P_{B}+w_{1} w_{2}\left(C_{B}+c\right)+w_{1} k+1\right),
\end{aligned}
$$

$$
\frac{\partial \pi^{2}\left(P_{A}, P_{B}\right)}{\partial P_{B}^{2}}=-2 w_{1} w_{2}\left(a-b P_{A}\right)<0 .
$$

Therefore, $\pi$ is a convex function of $P_{B}$. The proofs in II and III are the same as those of area I. 
Theorem 8. The process of the Stackelberg-merger game is divided into three situations according to areas I, II, III. There exists only one optimal pricing strategy for Stackelberg-merger game in each situation.

Proof. (i) In area I, $P_{B} \leq P_{A}$. the total profit of the SC can be got from the sum of (10):

$$
\begin{aligned}
\pi\left(P_{A}, P_{B}\right)= & \left(a-b P_{A}\right)\left(w_{1}\left(k-w_{2} P_{B}\right)+1\right) \\
& \times\left(P_{B}-C_{B}-c\right)-C_{g} .
\end{aligned}
$$

Base on Theorem 7 and Step 1, the optimal value $P_{B}$ of $\pi$ can be obtained as follows:

$$
P_{B}^{*}=\frac{\left(1+w_{1} k\right)}{\left(2 w_{1} w_{2}\right)}+\frac{\left(C_{B}+c\right)}{2} .
$$

The value of $w_{2}$ is so small that the value of $P_{B}{ }^{*}$ is greater than the maximum value of $P_{A 1}$. Considering $P_{B} \leq P_{A}$ and Theorem 7 , the maximum value of $\pi$ can be obtained in the condition that $P_{B}=P_{A}$, and then (21) can be expressed as follows:

$$
\pi\left(P_{A}\right)=\left(a-b P_{A}\right)\left(w_{1}\left(k-w_{2} P_{A}\right)+1\right)\left(P_{A}-C_{B}-c\right)-C_{g} .
$$

From $\partial \pi / \partial P_{A}=0$, the two values of $P_{A}$ can be got. One solution is so small that it cannot match the situation, and it is cast out. The other is the optimal value of $P_{A}$. So there is only one solution for area I (from Theorem 9).

(ii) In area II, the total profit of SC can be got from the sum of (11):

$$
\begin{aligned}
\pi\left(P_{A}, P_{B}\right)=(a & \left.-b P_{A}\right) \\
\times & \left\{\left(w_{3}\left(k w_{4} P_{A}-P_{B}\right)+w_{1}\left(k-w_{2} P_{B}\right)\right)\right. \\
\quad & \left(P_{B}-C_{B}-c\right) \\
& \left.+\left(1-w_{3}\left(k w_{4} P_{A}-P_{B}\right)\right)\left(P_{A}-C_{A}-c\right)\right\}-C_{g} .
\end{aligned}
$$

Based on Theorem 7 and Step 1, the optimal reaction $P_{B}\left(P_{A}\right)$ of $\pi$ can be obtained as follows:

$$
\begin{aligned}
P_{B}\left(P_{A}\right) & \\
& =\frac{\left(w_{3}\left(k w_{4}+1\right) P_{A}+w_{1} w_{2}\left(C_{B}+c\right)+w_{1} k+w_{3} C_{A}\right)}{2\left(w_{1} w_{2}+w_{3}\right)} .
\end{aligned}
$$

From Step 2, $P_{A}{ }^{*}$ can be got, and the expression of $P_{A}{ }^{*}$ is so long that it could not be written down here.

From Step 3, the optimal value of $P_{B}$ for $\pi$ can be obtained.

(iii) In area III, the total profit of SC can be derived from the sum of (12):

$$
\begin{aligned}
\pi\left(P_{A}, P_{B}\right)= & \left(a-b P_{A}\right) \\
& \times\left(w_{1}\left(k-w_{2} P_{B}\right)\left(P_{B}-C_{B}-c\right)+P_{A}-C_{A}-c\right) .
\end{aligned}
$$

Based on Theorem 7 and Step 1, the optimal value of $P_{B}$ for $\pi$ can be obtained as follows:

$$
P_{B}^{*}=\frac{k}{2 w_{2}}+\frac{\left(C_{B}+c\right)}{2} .
$$

From Step 2, the optimal of $P_{A}$ for $\pi$ can be obtained as follows:

$$
\begin{aligned}
P_{A}^{*}= & \frac{a}{2 b} \\
& -\frac{\left(w_{1}\left(k-\left(C_{B}+c\right)\right)\left(k-w_{2}\left(C_{B}+c\right)\right)-4\left(C_{A}+c\right)\right)}{8} .
\end{aligned}
$$

Theorem 9. In area $I$, the optimal value of $P_{A}$ is the only one valid solution, which matches the real situation.

Proof. In area I, from $\partial \pi\left(P_{A}\right) / \partial P_{A}=0$,

$$
\begin{aligned}
& 3 b w_{1} w_{2} P_{A}^{2}-\left(2 w_{1} w_{2} a+b\left(C_{B}+c\right)\left(w_{1} w_{2}\right)\right) \\
& +\left(w_{1} k+1\right)\left(a-b\left(C_{B}+c\right)\right)+w_{1} w_{2} a\left(C_{B}+c\right)=0
\end{aligned}
$$

Two $P_{A}$ can be got from (31):

$$
P_{A}=\frac{\left(\left(2 w_{1} w_{2} a+b\left(C_{B}+c\right)\left(w_{1} w_{2}-1\right)\right) \pm \sqrt{F}\right)}{\left(6 b w_{1} w_{2}\right)},
$$

where

$$
\begin{aligned}
F= & \left(2 w_{1} w_{2} a+b\left(C_{B}+c\right)\left(w_{1} w_{2}\right)\right)^{2} \\
& -12 b w_{1} w_{2}\left\{\left(w_{1} k+1\right)\left(a-b\left(C_{B}+c\right)\right)+w_{1} w_{2} a\left(C_{B}+c\right)\right\} .
\end{aligned}
$$

In (32), $P_{A}$ with the negative value of sqrt $F$ is

$$
\begin{aligned}
P_{A}^{1} & =\frac{\left(\left(2 w_{1} w_{2} a+b\left(C_{B}+c\right)\left(w_{1} w_{2}-1\right)\right)-\sqrt{F}\right)}{\left(6 b w_{1} w_{2}\right)} \\
& \approx \frac{a}{(3 b)}+\frac{\left(C_{B}+c\right)}{6}-\frac{1}{\left(6 w_{1} w_{2}\right)}-\frac{\sqrt{F}}{\left(6 b w_{1} w_{2}\right)},
\end{aligned}
$$

where

$$
\frac{\sqrt{F}}{\left(6 b w_{1} w_{2}\right)} \approx \frac{a}{(3 b)}+\frac{\left(C_{B}+c\right)}{6}-\frac{1}{\left(6 w_{1} w_{2}\right)} .
$$

Substituting (35) in (36),

$$
P_{A}^{1} \approx 0<C_{B}+c .
$$

It is obvious that the solution cannot match the real situation, and it was cast out. Therefore, the optimal value of $P_{A}$ is the only one valid solution, which matches the real situation.

The key of the Stackelberg-merger game is how to distribute the total profit between manufacturer and retailer. Using Nash bargaining model can coordinate profit of each entity [16], and the study of this field needs to go further. 
TABLE 2: The effect of RP cost changing in Stackelberg-Nash game $(k=1.2)$.

\begin{tabular}{|c|c|c|c|c|c|c|}
\hline \multirow{2}{*}{ Case result } & \multicolumn{3}{|c|}{$k=1.2 \quad C_{B}=220$} & \multicolumn{3}{|c|}{$k=1.2 \quad C_{B}=250$} \\
\hline & $P_{B} \leq P_{A}$ & $P_{A}<P_{B}<P_{B 0}$ & $P_{B 0}<P_{B}<P_{B 1}$ & $P_{B} \leq P_{A}$ & $P_{A}<P_{B}<P_{B 0}$ & $P_{B 0}<P_{B}<P_{B 1}$ \\
\hline$f_{A}$ & & 284.3316 & 266.0267 & & 286.2994 & 267.7067 \\
\hline$g_{A}$ & & 104.3316 & 86.0267 & & 106.2994 & 87.7067 \\
\hline$f_{B}$ & & 371.5171 & 540.0000 & & 393.8308 & 560.0000 \\
\hline$g_{B}$ & & 171.5171 & 340.0000 & & 163.8308 & 330.0000 \\
\hline$P_{A}$ & 365.9493 & 388.6632 & 352.0533 & 381.8052 & 392.5987 & 355.4133 \\
\hline$P_{B}$ & 365.9493 & 543.0343 & 880.0000 & 381.8052 & 557.6615 & 890.0000 \\
\hline$T$ & 268.1014 & 222.6737 & 295.8933 & 236.3896 & 214.8025 & 289.1733 \\
\hline$\Delta T$ & 178.8881 & 117.0312 & 75.7487 & 154.7302 & 110.3807 & 71.7150 \\
\hline$T_{s}$ & 268.1014 & 29.6436 & 0 & 236.3896 & 13.1986 & 0 \\
\hline$T_{A}$ & 0 & 193.0301 & 295.8933 & 0 & 201.6040 & 289.1733 \\
\hline$T_{B}$ & 536.2027 & 252.3172 & 295.8933 & 472.7791 & 228.0011 & 289.1733 \\
\hline$\pi_{r}$ & & 38502 & 43776 & & 35173 & 41811 \\
\hline$\pi_{m}$ & & 28502 & 33776 & & 25173 & 31811 \\
\hline$\pi$ & 46298 & 67005 & 77553 & 33729 & 60346 & 73621 \\
\hline
\end{tabular}

TABLE 3: The effect of RP cost changing in Stackelberg-Nash decision $(k=1.4)$.

\begin{tabular}{|c|c|c|c|c|c|c|}
\hline \multirow{2}{*}{ Case result } & \multicolumn{3}{|c|}{$k=1.4 \quad C_{B}=220$} & \multicolumn{3}{|c|}{$k=1.4 \quad C_{B}=250$} \\
\hline & $P_{B} \leq P_{A}$ & $P_{A}<P_{B}<P_{B 0}$ & $P_{B 0}<P_{B}<P_{B 1}$ & $P_{B} \leq P_{A}$ & $P_{A}<P_{B}<P_{B 0}$ & $P_{B 0}<P_{B}<P_{B 1}$ \\
\hline$f_{A}$ & & 279.2893 & 253.4637 & & 281.5733 & 255.4993 \\
\hline$g_{A}$ & & 99.2893 & 73.4637 & & 101.5733 & 75.4993 \\
\hline$f_{B}$ & & 399.1822 & 606.6667 & & 422.1999 & 626.6667 \\
\hline$g_{B}$ & & 199.1822 & 406.6667 & & 192.1999 & 396.6667 \\
\hline$P_{A}$ & 366.3029 & 378.5785 & 326.9000 & 382.0862 & 383.1467 & 331.0000 \\
\hline$P_{B}$ & 366.3029 & 598.3644 & 1013.3000 & 382.0862 & 614.3999 & 1023.3000 \\
\hline$T$ & 267.3943 & 242.8429 & 346.1452 & 232.8276 & 233.7066 & 338.0030 \\
\hline$\Delta T$ & 221.1238 & 155.7372 & 107.0742 & 192.0417 & 146.8800 & 101.8516 \\
\hline$T_{s}$ & 267.3943 & 73.1396 & 0 & 235.8276 & 54.7557 & 00 \\
\hline$T_{A}$ & 0 & 169.7034 & 346.1452 & 0 & 178.9509 & 338.0030 \\
\hline$T_{B}$ & 534.7886 & 315.9825 & 346.1452 & 471.6552 & 288.4624 & 338.0030 \\
\hline$\pi_{r}$ & & 54466 & 59910 & & 49319 & 57120 \\
\hline$\pi_{m}$ & & 44466 & 49910 & & 39319 & 47120 \\
\hline$\pi$ & 51701 & 98933 & 109820 & 37958 & 88639 & 104250 \\
\hline
\end{tabular}

\section{Numerical Simulation}

5.1. Stackelberg-Nash Game Simulation. Look at mobile phone, for example. There are two types of mobile phone $A$ and $B$ in the same market, and $B$ is the renewal product of $A$. The related parameters' values are as follows: $a=1000, b=2$, $w_{1}=0.8, w_{2}=0.001, w_{3}=0.008, w_{4}=1.2, C_{A}=200$. Table 2 shows the effect of the change of RP cost on price, demand, and profits of both products in the condition $k=1.2$. Table 3 shows the effect in the condition $k=1.4$. From Tables 2 and 3 , the effect of renewal rate $k$ on price, demand, and profits can be concluded.

From numerical simulation in Tables 2 and 3, the following conclusions can be drawn.

(1) There is no Stackelberg-Nash equilibrium in area I, and so Stackelberg-merger is used to simulate this situation. The total profit in this situation is the optimal profit of area I. Obviously, the optimal total profit of area I is less than those of areas II and III. Therefore, area $\mathrm{I}\left(P_{B}=P_{A}\right)$ is not the optimal area of Stackelberg-Nash game. The rational manufacturer and retailer could not select area I.

(2) For all parameter conditions, the profits of manufacturer, retailer, and the total profit of SC in area III are greater than those of area II (in area II, there is a little difference between the price of RP and OP). Because the manufacturer and retailer are rational, the final decision-making result $\left(P_{A}, P_{B}\right)$ of the whole SC should be in area III. In this condition, OP is set at a low price (break-even sales), while RP is priced at a high level (the price differential between $\mathrm{RP}$ and OP is huge); that is to say, RP comes onto the market at a high price, and OP comes onto the market at a low price, which leads to larger market 
TABLE 4: The effect of RP cost changing in Stackelberg-merger model $(k=1.2)$.

\begin{tabular}{|c|c|c|c|c|c|c|}
\hline \multirow{2}{*}{ Case result } & \multicolumn{3}{|c|}{$k=1.2 \quad C_{B}=220$} & \multicolumn{3}{|c|}{$k=1.2 \quad C_{B}=250$} \\
\hline & $P_{B} \leq P_{A}$ & $P_{A}<P_{B}<P_{B 0}$ & $P_{B 0}<P_{B}<P_{B 1}$ & $P_{B} \leq P_{A}$ & $P_{A}<P_{B}<P_{B 0}$ & $P_{B 0}<P_{B}<P_{B 1}$ \\
\hline$P_{A}$ & 365.9493 & 346.2141 & 267.8400 & 381.8052 & 346.2141 & 273.5100 \\
\hline$P_{B}$ & 365.9493 & 458.5284 & 720.0000 & 381.8052 & 473.5284 & 735.0000 \\
\hline$T$ & 268.1014 & 307.5718 & 464.3200 & 236.3896 & 307.5718 & 452.9800 \\
\hline$\Delta T$ & 178.8881 & 182.4446 & 178.2989 & 154.7302 & 178.7538 & 168.5086 \\
\hline$T_{s}$ & 268.1014 & 98.4720 & 0 & 236.3896 & 61.5634 & 0 \\
\hline$T_{A}$ & 0 & 209.0998 & 464.3200 & 0 & 246.0084 & 452.9800 \\
\hline$T_{B}$ & 536.2027 & 406.0438 & 464.3200 & 472.7791 & 369.1352 & 452.9800 \\
\hline$\pi$ & 46298 & 87780 & 107800 & 33729 & 79981 & 102600 \\
\hline
\end{tabular}

TABLE 5: The effect of RP cost changing in Stackelberg-merger model $(k=1.4)$.

\begin{tabular}{|c|c|c|c|c|c|c|}
\hline \multirow{2}{*}{ Case result } & \multicolumn{3}{|c|}{$k=1.4 \quad C_{B}=220$} & \multicolumn{3}{|c|}{$k=1.4 \quad C_{B}=250$} \\
\hline & $P_{B} \leq P_{A}$ & $P_{A}<P_{B}<P_{B 0}$ & $P_{B 0}<P_{B}<P_{B 1}$ & $P_{B} \leq P_{A}$ & $P_{A}<P_{B}<P_{B 0}$ & $P_{B 0}<P_{B}<P_{B 1}$ \\
\hline$\overline{P_{A}}$ & 366.3029 & 346.2141 & 225.4400 & 382.0862 & 346.2141 & 232.3100 \\
\hline$P_{B}$ & 366.3029 & 505.3881 & 820.0000 & 382.0862 & 520.3881 & 835.0000 \\
\hline$T$ & 267.3943 & 307.5718 & 549.1200 & 232.8276 & 307.5718 & 535.3800 \\
\hline$\Delta T$ & 221.1238 & 220.1259 & 254.7917 & 192.0417 & 216.4351 & 241.9918 \\
\hline$T_{s}$ & 267.3943 & 187.6227 & 0 & 235.8276 & 150.7141 & 0 \\
\hline$T_{A}$ & 0 & 119.491 & 549.1200 & 0 & 156.8577 & 535.3800 \\
\hline$T_{B}$ & 534.7886 & 495.1945 & 549.1200 & 471.6552 & 458.2859 & 535.3800 \\
\hline$\pi$ & 51701 & 123350 & 150770 & 37958 & 111730 & 143320 \\
\hline
\end{tabular}

scale and wider influence on product. In this case, even if RP is set at a high price, the shift demand and the increment demand of market are proportional to the size of OP scale, so high price has less influence on market demand. But the profit of unit renewal product rises substantially with its price increase. In this situation, RP comes onto the market with a great price differential, and so the profits of all products are the highest; that is, area III is the optimal pricing area of supply chain.

(3) The profit of retailer in areas II and III is greater than that of manufacturer, mainly because the retailer is a direct participator of market, and it reacts very rapidly when the demand of market changes. Therefore, the retailer can adjust the process of decision making in the fastest time to increase revenue and reduce losses. The manufacturer's reaction to the market relies on the retailer decision making information's transmission, and there is a delay in the transmission process. Accordingly, the manufacturer's profit is less than that of the retailer.

(4) With the cost of RP increasing, the profits of the manufacturer, retailer, and total will decrease.

(5) If the cost of RP is constant, with product renewal rate $k$ increasing, the profits of the manufacturer, retailer and total will increase.

5.2. Stackelberg-Merger Game Simulation. The parameters are the same as Section 5.1. With the changing of product renewal rate or the cost of RP, the variety of price, demand, and profits are shown in Tables 4 and 5.

From numerical simulation in Tables 4 and 5, the following conclusions can be drawn:

(i) The profits in areas II and III of Stackelberg-merger game are greater than those in area I, so the Stackelberg-merger pricing strategy would not be in area I. The situation is consistent with StackelbergNash game.

(ii) If OP adopts low-price strategy, and RP adopts highprice strategy, the total profit will be maximum value; in other words, area III is the optimal merger pricing area for the SC. The situation and its reason are the same as those of Stackelberg-Nash game.

(iii) If the cost of RP is constant, with the product renewal rate increasing, the total profit will increase obviously.

(iv) With the cost of RP increasing, the total profit will decrease.

From the analysis of Sections 5.1 and 5.2, the following can be drawn: (i) Both two kinds of decision-making processes are consistent with each other in profits, cost, and product renewal rate changing (with the cost of $\mathrm{RP}$ increasing, profits decrease, with product renewal rate increasing, profits increase). (ii) Both cases obtain the optimal profits in area III; in other words, the RP which comes onto the market has a great price differential with OP. (iii) Compared with Stackelberg-Nash game, the price in Stackelberg-merger 
game is lower while the profits are more, so Stackelbergmerger decision making is virtually a kind of win-win situation for enterprises and market.

\section{Conclusion}

This paper studied the process of product renewal. A market shift model of RP was built on incremental function and shift function. Based on the model, Stackelberg-Nash game model and Stackelberg-merger game model for RP in SC were built, and their theoretical analysis was carried out. The following conclusions can be drawn. (i) In both two models, the increase of RP cost will make participants' profits and total profit decrease, while higher renewal rate will make the profits increase at the same margin cost. (ii) Manufacturer and retailer obtain the optimal profits in area III; in other words, the way of the optimal decision making in SC is that RP comes onto the market with a great price differential with OP. (iii) Compared with Stackelberg-Nash game model, Stackelbergmerger game model's pricing is lower and the profits are higher, which is actually a kind of win-win situation for enterprises and market.

In this paper, a part of premise conditions of the model was built on the basis of some rational hypothesis, and the model parameters need to be confirmed by real statistics data. These problems need to be further studied.

\section{Acknowledgment}

This work was supported by the National Natural Science Foundation of China (Grant no. 71002106).

\section{References}

[1] T. Dereli, A. Baykasoğlu, and G. Büyüközkan, "An affordable reverse engineering framework for innovative rapid product development," International Journal of Industrial and Systems Engineering, vol. 3, no. 1, pp. 31-37, 2008.

[2] M. Bourreau, P. Dogan, and M. Manant, "Modularity and product innovation in digital markets," Review of Network Economics, vol. 6, no. 2, pp. 175-193, 2007.

[3] C. Ju and L. Xiao, "Model of product lifecycle evaluation based on context knowledge," Journal of Computational Information Systems, vol. 4, no. 4, pp. 1753-1760, 2008.

[4] F. C. Wan, D. W. Wang, and P. Y. Chen, "Correlative product combinatorial introduction model," Control Theory \& Applications, vol. 21, no. 2, pp. 257-260, 2004.

[5] P. Huo, The analysis of the R\&D strategic technology in supply chain based on game theory, Postdoctoral study paper of Tsinghua University, 2003.

[6] P. I. Bass and F. M. Bass, "Diffusion of technology generations: a model of adoption and repeat sales," http://www.bassbasement.org/F/N/BBDL/Bass\%20and\%20Bass\%202001.pdf.

[7] P. I. Bass and F. M. Bass, "IT waves: two completed generational diffusion models," 2004, http://www.bassbasement .org/F/N/BBDL/Bass\%20and\%20Bass\%202004.pdf.

[8] L. Wu, R. De Matta, and T. J. Lowe, "Updating a modular product: how to set time to market and component quality," IEEE Transactions on Engineering Management, vol. 56, no. 2, pp. 298-311, 2009.
[9] C. T. Druehl, G. M. Schmidt, and G. C. Souza, "The optimal pace of product updates," European Journal of Operational Research, vol. 192, no. 2, pp. 621-633, 2009.

[10] Y. Huang and F. Ling, "Study on the tracks of product update of ICT enterprises," in Proceedings of the 2nd International Conference on E-Business and E-Government (ICEE '11), pp. 6400-6403, May 2011.

[11] E. Koca, G. C. Souza, and C. T. Druehl, "Managing product rollovers," Decision Sciences, vol. 41, no. 2, pp. 403-423, 2010.

[12] X. W. Quan, "The pricing game of updated and existing products," Acta Scientiarum Naturalium Universitatis Nankaiensis, vol. 44, no. 3, pp. 1-7, 2011.

[13] Y. Luo and B. S. Tu, "Supply chain decision making model for product updating based on game theory," Computer Integrated Manufacturing Systems, vol. 18, no. 9, pp. 2067-2075, 2012.

[14] J. Wei, Pricing decisions and inventory decisions for innovative product and substitutable product [Ph.D. dissertation], Control Engineering of Nankai University, Tianjin, China, 2007.

[15] S. Zhu, Microeconomics, Beijing University, 2nd edition, 2001.

[16] D. Mou, Decision analysis for supply chain management based on game theory [Ph.D. dissertation], Control Engineering of Nankai University, Tianjin, China, 2003. 


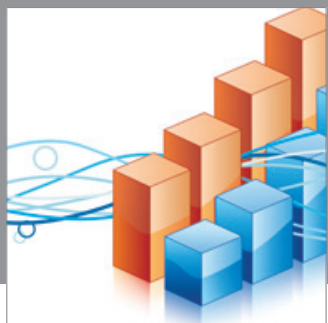

Advances in

Operations Research

mansans

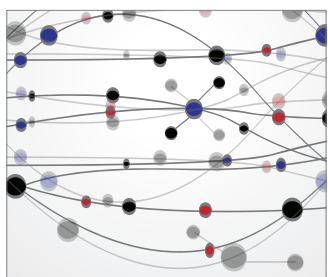

The Scientific World Journal
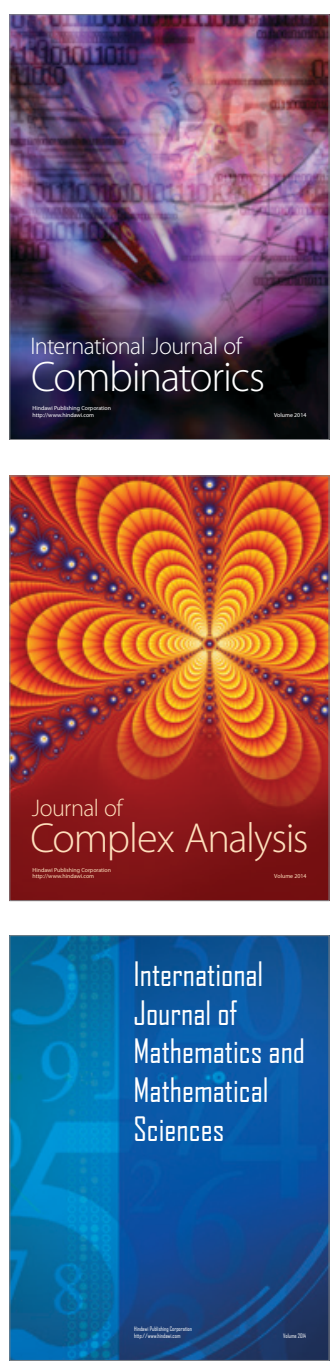
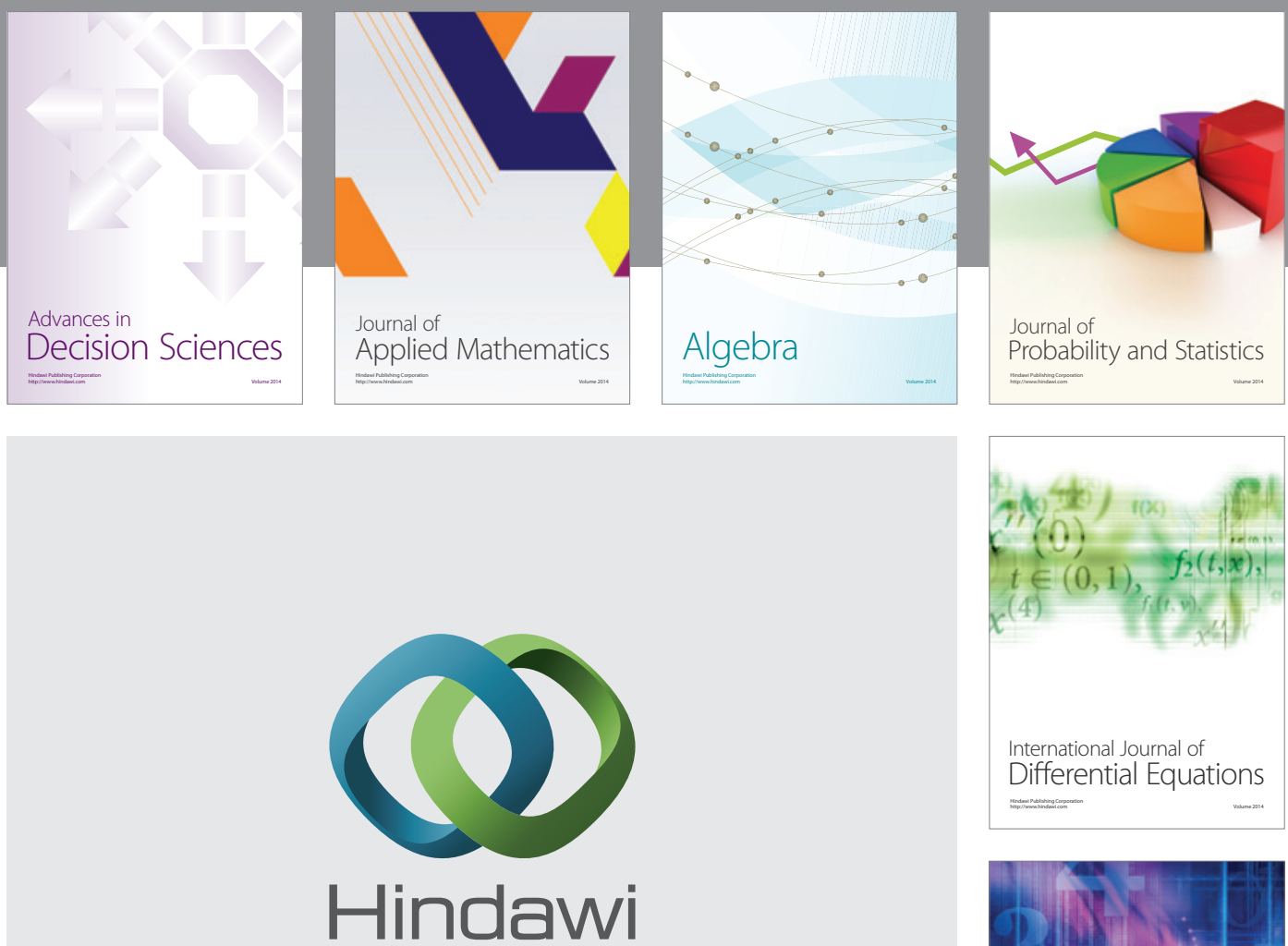

Submit your manuscripts at http://www.hindawi.com
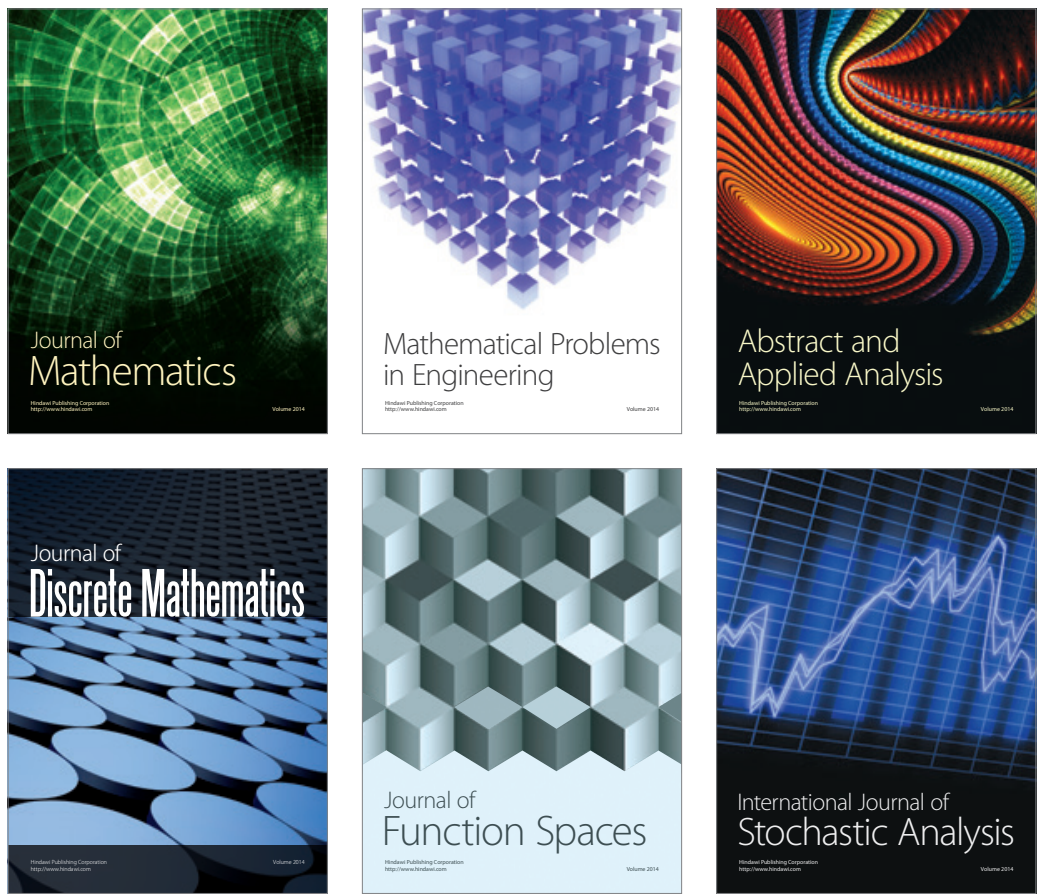

Journal of

Function Spaces

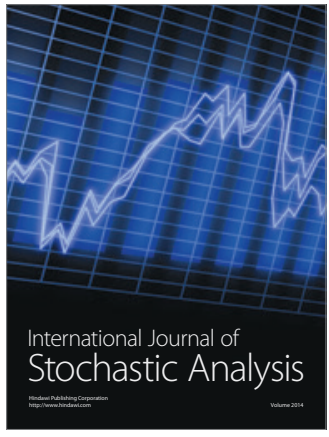

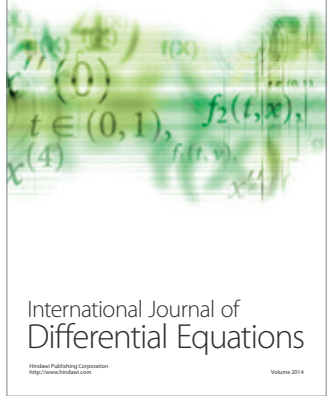
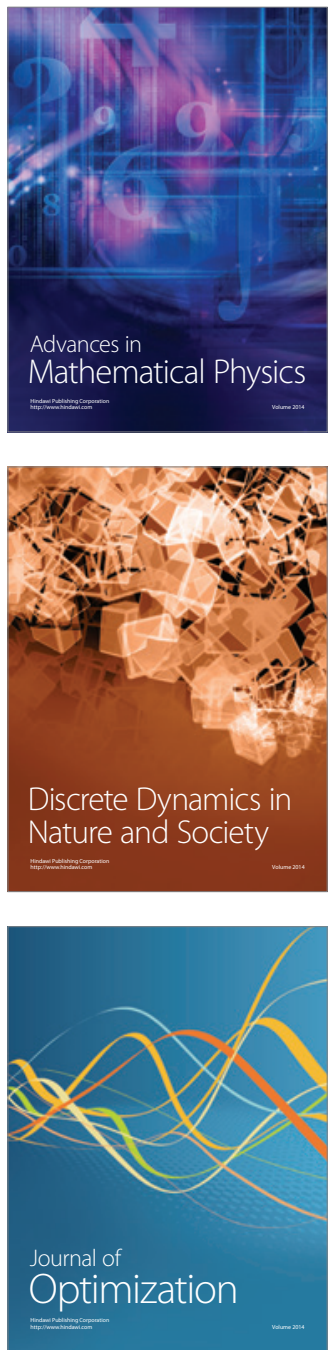